\title{
Extracorporeal membrane oxygenation for accidental deep hypothermia-current challenges and future perspectives
}

\author{
Piotr Mazur ${ }^{1,2}$, Sylweriusz Kosiński ${ }^{3}$, Paweł Podsiadło ${ }^{4}$, Anna Jarosz ${ }^{1}$, Roman Przybylski ${ }^{1}$, \\ Radosław Litiwnowicz ${ }^{1}$, Jacek Piątek ${ }^{1}$, Janusz Konstanty-Kalandyk ${ }^{1,2}$, Robert Gałązkowski ${ }^{5}$, \\ Tomasz Darocha ${ }^{6}$
}

${ }^{1}$ Department of Cardiovascular Surgery and Transplantology, John Paul II Hospital, Cracow, Poland; ${ }^{2}$ Institute of Cardiology, Jagiellonian University Medical College, Cracow, Poland; ${ }^{3}$ Faculty of Health Sciences, Jagiellonian University Medical College, Cracow, Poland; ${ }^{4}$ Emergency Medicine Department, Jan Kochanowski University, Kielce, Poland; ${ }^{5}$ Department of Emergency Medical Services, Medical University of Warsaw, Warsaw, Poland; ${ }^{6}$ Department of Anesthesiology and Intensive Care Medicine, Medical University of Silesia, Katowice, Poland

Correspondence to: Tomasz Darocha, MD, PhD. Department of Anesthesiology and Intensive Care Medicine, Medical University of Silesia, Katowice, Poland. Email: tomekdarocha@wp.pl.

\begin{abstract}
The incidence of accidental hypothermia (core temperature $\leq 35^{\circ} \mathrm{C}$ ) is difficult to estimate, as the affected population is heterogeneous. Both temperature and clinical presentation should be considered while determining severity, which is difficult in a prehospital setting. Extracorporeal rewarming is advocated for all Swiss Staging System class IV (hypothermic cardiac arrest) and class III (hypothermic cardiac instability) patients. Veno-arterial extracorporeal membrane oxygenation (ECMO) is the method of choice, as it not only allows a gradual, controlled increase of core body temperature, but also provides respiratory and hemodynamic support during the unstable period of rewarming and reperfusion. This poses difficulties with the coordination of patient management, as usually only cardiac referral centers can deliver such advanced treatment. Further special considerations apply to subgroups of patients, including drowning or avalanche victims. The principle of ECMO implantation in severely hypothermic patients is no different from any other indication, although establishing vascular access in a timely manner during ongoing resuscitation and maintaining adequate flow may require modification of the operating technique, as well as aggressive fluid resuscitation. Further studies are needed in order to determine the optimal rewarming rate and flow that would favor brain and lung protection. Recent analysis shows an overall survival rate of $40.3 \%$, while additional prognostic factors are being sought for determining those patients in whom the treatment is futile. New cannulas, along with ready-to-use ECMO sets, are being developed that would enable easy, safe and efficient out-reach ECMO implantation, thus shortening resuscitation times. Moreover, national guidelines for the management of accidental hypothermia are needed in order that all patients that would benefit from extracorporeal rewarming would be provided with such treatment. In this perspective article, we discuss burning problems in ECMO therapy in hypothermic patients, outlining the important research goals to improve the outcomes.
\end{abstract}

Keywords: Extracorporeal membrane oxygenation (ECMO); accidental hypothermia; cardiac arrest; rewarming

Submitted Aug 27, 2018. Accepted for publication Oct 25, 2018.

doi: $10.21037 /$ acs.2018.10.12

View this article at: http://dx.doi.org/10.21037/acs.2018.10.12

\section{Introduction}

Accidental hypothermia (core temperature $\leq 35^{\circ} \mathrm{C}$ ) is a deleterious condition carrying a high risk of mortality which, with appropriate measures, can be successfully treated (1). In the United States alone, accidental hypothermia causes almost 1,500 deaths annually (1). Although accidental hypothermia as a medical condition displays a tendency to occur in colder climates, with many 
reports coming from northern countries such as Norway $(2,3)$, Canada (4); Alpine regions of Switzerland (5), Austria (6) or France (7); and regions at lower altitudes with a moderate climate, such as The Netherlands (8), Poland (9), France (10), Austria (Lower Austria) (11), Denmark (12) or Japan (13), it is not confined to any geographical area, as evidenced by cases reported even from Australia (14).

The incidence of accidental hypothermia appears to be underestimated, mainly due to diagnostic problems. Although the gold standard is to measure the patient's core temperature, such measurement is difficult to conduct during rescue operations. In the prehospital setting, a clinical staging scheme (Swiss Staging System; HT) is employed (1). Based on recent recommendations, hypothermic patients with cardiac instability at stage HT III (core body temperature $<28^{\circ} \mathrm{C}$ ) and HT IV (hypothermic cardiac arrest) are candidates for rewarming using extracorporeal life support (ECLS) with cardiopulmonary bypass $(\mathrm{CPB})$ or extracorporeal membrane oxygenation (ECMO) (1,15-17).

The first reports on the use of CPB for extracorporeal rewarming in accidental hypothermia date back to 1967 (18). Successful ECLS-aided rewarming has been reported from temperatures as low as $13.7{ }^{\circ} \mathrm{C}(\mathrm{CPB})(19)$, and a period as long as $6 \mathrm{~h} 45 \mathrm{~min}$ after hypothermic cardiac arrest (with ventricular fibrillation) (ECMO) (20). Today, a growing body of evidence supports the notion that ECMO in its veno-arterial (VA) configuration is a better treatment for hypothermic, unstable or arrested patients (21). An increasing number of institutions consider VA-ECMO as the first-line of treatment due to the possibility of prolonged support and a decreased incidence of severe cardiorespiratory instability $(22,23)$, also in the pediatric population (24).

The patient spectrum will differ from institution to institution, however, making the population very inhomogeneous. Depending on the location of the reporting hospital, therefore, the majority of patients may be avalanche victims or mountaineers $(5,6)$ - mostly fairly young, with no significant underlying medical problems, but with potential trauma and asphyxiation; patients from urban populations $(8,10,11,13)$-often intoxicated, frequently suffering from comorbidities; or drowning victims $(10,12)$ at a high risk of primary asphyxia. The outcomes in these populations are varied. The authors represent a Severe Hypothermia Treatment Center (22), which, due to its geographical location, manages a variety of hypothermic patients, ranging from urban cases, through drowning victims, to mountain accident victims.

\section{Guidelines and coordination}

The specificity of accidental hypothermia management is that it always takes place in an acute setting. Due to a paucity of data-virtually no randomized trials have been conducted to date-strong evidence endorsing any strategy is lacking, although the general consensus is that ECLS is the gold standard in hypothermic patients with cardiac arrest (16), with VA-ECMO being the preferred method due to its rapid availability, lower heparinization requirement (as compared with CPB), and possibility of prolonged support (15). Several algorithms have been developed that mandate the use of ECLS for rewarming, including the Bernese Hypothermia Algorithm (5) and the algorithm endorsed by the International Commission for Mountain Emergency Medicine (15). ECLS is indicated in HT IV and HT III in unstable non-arrested patients (understood as those presenting with life-threatening arrhythmia, hypotension with a systolic blood pressure of $<90 \mathrm{mmHg}$, respiratory failure or refractory acidosis) (15). The coordination of the rescue effort and arrangement of the rewarming procedure is complex, and usually rests on the intensivist/ anesthesiologist or emergency physician. In the context of the complexity of the whole process (e.g., coordination of mountain rescue services; emergency medical services with an automated chest compression device; ground and/or air transportation; and, finally, operating room personnel with cardiac surgeons or an out-reach ECMO team) the existence of accidental hypothermia treatment centers and coordinators seems reasonable in high prevalence areas.

Many cases are likely mistreated worldwide due to a lack of information, coordination and cooperation. Higher-level regulations in this regard are urgently needed-the ECLS management of severe accidental hypothermia should be regulated (and funded) on a national governmental level. A hypothermia coordinator, who is trained in decision making, may support physicians treating hypothermic patients and help in deciding whether ECLS is indicated (9). Decisionmaking is oftentimes difficult, based on fragmented data, while our clinical practice shows that there are very few straightforward cases. Apart from the fact that the clinical presentation of severe hypothermia is difficult to distinguish from the clinical signs of death (posing a problem for prehospital services who have not been trained), patients often present complex cases (e.g., trauma and asphyxiation in avalanche victims or intoxication and underlying conditions in urban cases). What is more, there may be a need for the simultaneous ECLS treatment of multiple 
patients (12), where inter-institutional coordination is crucial. In this perspective article, we discuss pertinent procedural and post-procedural issues in ECMO therapy in hypothermic subjects. We also discuss current outcomes of extracorporeal resuscitation in severe hypothermia and outline the important research goals to improve them.

\section{Technical and practical considerations}

VA-ECMO offers cardiac support, enabling treatment of arrested patients, with the highest achievable rewarming rate (up to $6^{\circ} \mathrm{C} / \mathrm{h}$ ) and a wide flow span (1). There are significant differences in rewarming strategies among reports, while the optimal speed of rewarming and ECLS flow are still to be established. Animal studies on a porcine model of accidental deep hypothermia with cardiac arrest revealed that low ECLS flow does not attenuate post-rewarming pulmonary lesions, but decreases cardiac output (25). On the other hand, also in a porcine model, it has been shown that a high temperature delta between the core temperature and ECLS increases lung injury (26). A recent analysis by Saczkowski et al. revealed that a slower rate of ECLS rewarming is independently associated with an improved neurologic outcome (23). This is in line with what we know about rewarming from hypothermia during cardiac surgery (27).

On the other hand, it should be pointed out that in cardiac arrest or severe cardiorespiratory insufficiency, the priority is to move the patient out of the danger zone (below $28-30{ }^{\circ} \mathrm{C}$ ) as quickly as possible. In this range of core temperatures, the risk of spontaneous or iatrogenic $\mathrm{VF}$ is very high. Moreover, we do not know whether different types of hypothermia (acute, urban) require similar or different rewarming strategies. Future research should also answer the burning question of whether a patient suffering from cardiac arrest due to accidental hypothermia should be managed with mild therapeutic hypothermia in the postresuscitation period of ECMO as well. Severe peripheral complications can complicate ECMO when femoral access is used, with up to $20 \%$ of patients suffering from limb ischemia $(4,28)$. Although the use of distal perfusion cannulas is routine, implantation during cardiopulmonary resuscitation (CPR) in an arrested patient may be, and often is, challenging. Furthermore, in some ECMO centers, percutaneous ultrasound-guided placement of the arterial and venous cannulas is preferred (10). It is difficult to achieve adequate peripheral perfusion with this technique.

There is a need for new cannulas that permit parallel retrograde flow, enabling continuous peripheral extremity perfusion. Currently, new and virtually wall-less arterial cannulas are being studied that maintain bidirectional flow (29). Although such an approach would also simplify the circuit and the implantation procedure, their feasibility during CPR implantation in hypothermia is unknown. Our strategy is to bring the patient to the operating room and perform the procedure surgically, with as much asepticity as possible, even if the patient is in an extremely disheveled state. As ECMO therapy increases the risk of sepsis substantially (30), even if the implantation is elective, all efforts should be made to minimize this risk in the acute setting. The Bernese group suggest that in patients with suspected trauma, a focused assessment with sonography in trauma (FAST) should be performed simultaneously with the establishment of femoral access (5). Although better strategies in patients with suspected trauma are as yet unknown, a CT trauma scan prior to the onset of ECMO installation still seems a reasonable course of action. The major concern in extracorporeal rewarming in patients with traumatic injuries is the risk of heparinization-related bleeding.

It should be highlighted, however, that even severe poly-trauma seems not to be an absolute contraindication for ECLS-aided rewarming, as commonly believed. The heparin-coated circuits offer supplemental cardiopulmonary support for selected massively injured patients, both during CPR and while their primary injuries are being evaluated and treated $(31,32)$. On the other hand, a French group recently reported that ECMO implantation is already feasible during out-of-hospital CPR for refractory cardiac arrest, thus on-site (33). Another problem often met in severe hypothermic patients is volume depletion and the need for aggressive fluid replacement in order to maintain sufficient ECLS inflow. Unfortunately, microcirculatory derangement and diffuse endothelial damage "open the door" to extravasation and massive tissue edema that would necessitate emergency fasciotomies, laparotomies and/ or drainage to treat compartment syndromes. In addition, acute renal failure as a direct consequence of volume depletion and intraabdominal compartment syndrome is often seen in the post-resuscitation phase and requires renal replacement therapy.

\section{Post-rewarming issues}

Patients who have been successfully rewarmed still constitute a challenging group. Although a good proportion 
of hypothermic subjects treated with ECLS present good neurologic outcomes [Cerebral Performance Category (CPC) 1 or 2] (23), virtually all patients require attentive rehabilitation care while some will never recover fully. Thus, any ECMO center should be prepared for intensive early rehabilitation and be able to discharge the patient to a rehabilitation facility where the required skills are available. In some cases of urban hypothermia, physicians will face successfully rescued individuals with substance abuse issues, those who are homeless, and lack social support. Social services should participate in the management of such patients in order to obtain a good recovery, as well as solve reimbursement issues for those uninsured. Furthermore, some individuals will suffer from associated wound issues, not directly associated with ECLS (i.e., frostbite, ulcers etc.). Although the management of these problems is complicated and cost-consuming, they should be anticipated and considered beforehand. Despite the cost of ECMO rewarming and subsequent ICU stays being high, this was reported to be lower than in ECMO for other indications and intensive care treatment for other causes (34). Moreover, many rewarmed patients with good neurologic outcomes return to their vocational activity and social life, reducing the social burden of accidental hypothermia.

\section{Outcomes and prognostication}

The outcomes of ECMO treatment for severe accidental hypothermia with hemodynamic instability or cardiac arrest are encouraging to the point where the authors would suggest that any cardiac center equipped with ECMO should be involved in an ECLS hypothermia treatment program. A recent analysis of 44 observational studies and 40 case reports by Saczkowski et al. (23), comprising 658 patients with accidental hypothermia treated with ECLS, revealed an overall survival rate with a good neurologic outcome of $40.3 \%$ (23). This study indicated a higher rewarming rate, asphyxiation, and a higher serum potassium level as the negative independent predictors of good neurologic outcome, while the female gender seemed to be protective (23). The last factor is in striking alignment with the outcomes of the Præstø Fjord boating accident which occurred in Denmark in 2011 (12). Our data on 29 patients treated with VA ECMO in severe hypothermia (HT III and IV) revealed one-year survival of $45 \%$ (most patients with CPC 1) (34).

Notwithstanding, it is rather difficult to predict which patient will present with a favorable outcome, and in whom the treatment is futile. Most studies report high potassium levels $(>12 \mathrm{mmol} / \mathrm{L}$ in accidental hypothermia and $>8 \mathrm{mmol} / \mathrm{L}$ for hypothermia due to avalanche burial) as a predictor of poor outcome (35). A recent publication by Pasquier et al. presented the first outcome prediction tool for ECLS-treated patients with hypothermic cardiac arrest ("The HOPE score", developed based on international data from 286 patients) (35). The calculator (available online at: www.hypothermiascore.org) estimates one's chance of survival, considering age, gender, asphyxia, CPR duration, serum potassium level and temperature (35). Further answers with regard to outcome prediction are likely to be yielded by the International Hypothermia Registry (www. hypothermia-registry.org), in which the data of patients with accidental hypothermia are collected.

\section{Perspectives and research goals}

Hypothermia is associated with substantial fluid shifts with microvascular extravasation. Although hemodilution with ECMO may be beneficial, it is unknown whether different types of priming solutions may modulate the outcomes. While cerebral edema is a major concern, the effect of priming solution composition (crystalloids $v s$. colloids) has not been studied in subjects suffering from accidental hypothermia. A future goal is also the development of an ECMO circuit that would be easy to prime (a preprepared set with a one-click-to-prime type of technological solution), fail-safe and requiring no heparinization, in order that it could be easily set up and started in the setting of emergency department, without the need of perfusionist in attendance, with the aim of shortening the door-toECMO time, e.g., in cases of hypothermic cardiac arrest. New technologies are needed to extend the possible ECMO treatment duration with coated circuits, when no heparin is administered. On the other hand, considering the fact that a substantial subset of hypothermic patients may represent moribund trauma patients, circuits made with new materials that would be entirely anticoagulation-free should be developed. Nevertheless, even if ECMO is implanted in the emergency room, or even on-site (33) by an out-reach team, such patients should ideally be managed in the cardiac ICU of a multidisciplinary referral hospital.

\section{Conclusions}

Further studies are needed in order to provide evidencebased protocols for extracorporeal rewarming techniques 
in severe accidental hypothermia. Outcomes, however, are already very favorable, even in patients with even long resuscitation times for hypothermic cardiac arrest, thereby justifying the statement that every cardiac surgery center should be ready and available for ECLS rewarming in accidental deep hypothermia.

\section{Acknowledgements}

Funding: This study was funded by a grant from the Jagiellonian University, Cracow, Poland (No. K/ ZDS/007961 to P Mazur).

\section{Footnote}

Conflicts of Interest: The authors have no conflicts of interest to declare.

\section{References}

1. Brown DJ, Brugger H, Boyd J, et al. Accidental hypothermia. N Engl J Med 2012;367:1930-8.

2. Hilmo J, Naesheim T, Gilbert M. "Nobody is dead until warm and dead": prolonged resuscitation is warranted in arrested hypothermic victims also in remote areas--a retrospective study from northern Norway. Resuscitation 2014;85:1204-11.

3. Farstad M, Andersen KS, Koller ME, et al. Rewarming from accidental hypothermia by extracorporeal circulation. A retrospective study. Eur J Cardiothorac Surg 2001;20:58-64.

4. Vretenar DF, Urschel JD, Parrott JC, et al. Cardiopulmonary bypass resuscitation for accidental hypothermia. Ann Thorac Surg 1994;58:895-8.

5. Monika BM, Martin D, Balthasar E, et al. The Bernese Hypothermia Algorithm: a consensus paper on in-hospital decision-making and treatment of patients in hypothermic cardiac arrest at an alpine level 1 trauma centre. Injury 2011;42:539-43

6. Mair $\mathrm{P}$, Brugger $\mathrm{H}, \mathrm{Mair} \mathrm{B}$, et al. Is extracorporeal rewarming indicated in avalanche victims with unwitnessed hypothermic cardiorespiratory arrest? High Alt Med Biol 2014;15:500-3.

7. Debaty G, Moustapha I, Bouzat P, et al. Outcome after severe accidental hypothermia in the French Alps: A 10year review. Resuscitation 2015;93:118-23.

8. van der Ploeg GJ, Goslings JC, Walpoth BH, et al. Accidental hypothermia: rewarming treatments, complications and outcomes from one university medical centre. Resuscitation 2010;81:1550-5.

9. Jarosz A, Darocha T, Kosinski S, et al. Profound Accidental Hypothermia: Systematic Approach to Active Recognition and Treatment. ASAIO J 2017;63:e26-30.

10. Champigneulle B, Bellenfant-Zegdi F, Follin A, et al. Extracorporeal life support (ECLS) for refractory cardiac arrest after drowning: an 11-year experience. Resuscitation 2015;88:126-31.

11. Schober A, Sterz F, Handler C, et al. Cardiac arrest due to accidental hypothermia--a 20 year review of a rare condition in an urban area. Resuscitation 2014;85:749-56.

12. Wanscher M, Agersnap L, Ravn J, et al. Outcome of accidental hypothermia with or without circulatory arrest: experience from the Danish Praesto Fjord boating accident. Resuscitation 2012;83:1078-84.

13. Morita S, Inokuchi S, Yamagiwa T, et al. Efficacy of portable and percutaneous cardiopulmonary bypass rewarming versus that of conventional internal rewarming for patients with accidental deep hypothermia. Crit Care Med 2011;39:1064-8.

14. Dunne B, Christou E, Duff O, et al. Extracorporealassisted rewarming in the management of accidental deep hypothermic cardiac arrest: a systematic review of the literature. Heart Lung Circ 2014;23:1029-35.

15. Paal P, Gordon L, Strapazzon G, et al. Accidental hypothermia-an update : The content of this review is endorsed by the International Commission for Mountain Emergency Medicine (ICAR MEDCOM). Scand J Trauma Resusc Emerg Med 2016;24:111.

16. Truhlář A, Deakin CD, Soar J, et al. European Resuscitation Council Guidelines for Resuscitation 2015: Section 4. Cardiac arrest in special circumstances. Resuscitation 2015;95:148-201.

17. Zafren K, Giesbrecht GG, Danzl DF, et al. Wilderness Medical Society practice guidelines for the out-of-hospital evaluation and treatment of accidental hypothermia: 2014 update. Wilderness Environ Med 2014;25:S66-85.

18. Davies DM, Millar EJ, Miller IA. Accidental hypothermia treated by extracorporeal blood warming. Lancet 1967;1:1036-7.

19. Gilbert M, Busund R, Skagseth A, et al. Resuscitation from accidental hypothermia of 13.7 degrees $\mathrm{C}$ with circulatory arrest. Lancet 2000;355:375-6.

20. Kosinski S, Darocha T, Jarosz A, et al. The longest persisting ventricular fibrillation with an excellent outcome - 6h 45min cardiac arrest. Resuscitation 2016;105:e21-2.

21. Ruttmann E, Weissenbacher A, Ulmer H, et al. Prolonged 
extracorporeal membrane oxygenation-assisted support provides improved survival in hypothermic patients with cardiocirculatory arrest. J Thorac Cardiovasc Surg 2007;134:594-600.

22. Darocha T, Kosinski S, Jarosz A, et al. Severe Accidental Hypothermia Center. Eur J Emerg Med 2015;22:288-91.

23. Saczkowski RS, Brown DJA, Abu-Laban RB, et al. Prediction and risk stratification of survival in accidental hypothermia requiring extracorporeal life support: An individual patient data meta-analysis. Resuscitation 2018;127:51-7.

24. Scaife ER, Connors RC, Morris SE, et al. An established extracorporeal membrane oxygenation protocol promotes survival in extreme hypothermia. J Pediatr Surg 2007;42:2012-6.

25. Debaty G, Maignan M, Perrin B, et al. Cardiopulmonary responses during the cooling and the extracorporeal life support rewarming phases in a porcine model of accidental deep hypothermic cardiac arrest. Scand J Trauma Resusc Emerg Med 2016;24:91.

26. Debaty G, Maignan M, Perrin B, et al. Deep Hypothermic Cardiac Arrest Treated by Extracorporeal Life Support in a Porcine Model: Does the Rewarming Method Matter? Acad Emerg Med 2016;23:665-73.

27. Engelman R, Baker RA, Likosky DS, et al. The Society of Thoracic Surgeons, The Society of Cardiovascular Anesthesiologists, and The American Society of ExtraCorporeal Technology: Clinical Practice Guidelines for Cardiopulmonary Bypass--Temperature Management During Cardiopulmonary Bypass. Ann Thorac Surg 2015;100:748-57.

28. Mohite PN, Fatullayev J, Maunz O, et al. Distal limb

Cite this article as: Mazur P, Kosiński S, Podsiadło P, Jarosz A, Przybylski R, Litiwnowicz R, Piątek J, Konstanty-Kalandyk J, Gałązkowski R, Darocha T. Extracorporeal membrane oxygenation for accidental deep hypothermia-current challenges and future perspectives. Ann Cardiothorac Surg 2019;8(1):137-142. doi: 10.21037/acs.2018.10.12 perfusion: Achilles' heel in peripheral venoarterial extracorporeal membrane oxygenation. Artif Organs 2014;38:940-4.

29. Berdajs D, Ferrari E, Michalis A, et al. New prototype of femoral arterial SmartCannula with anterograde and retrograde flow. Perfusion 2011;26:271-5.

30. Raffa GM, Kowalewski M, Brodie D, et al. Meta-Analysis of Peripheral or Central ECMO in Postcardiotomy and Non-Postcardiotomy Shock. Ann Thorac Surg 2019;107:311-21.

31. Perchinsky MJ, Long WB, Hill JG, et al. Extracorporeal cardiopulmonary life support with heparin-bonded circuitry in the resuscitation of massively injured trauma patients. Am J Surg 1995;169:488-91.

32. Arlt M, Philipp A, Voelkel S, et al. Extracorporeal membrane oxygenation in severe trauma patients with bleeding shock. Resuscitation 2010;81:804-9.

33. Lamhaut L, Hutin A, Puymirat E, et al. A Pre-Hospital Extracorporeal Cardio Pulmonary Resuscitation (ECPR) strategy for treatment of refractory out hospital cardiac arrest: An observational study and propensity analysis. Resuscitation 2017;117:109-17.

34. Kosiński S, Darocha T, Czerw A, et al. Cost-utility of extracorporeal membrane oxygenation rewarming in accidentally hypothermic patients-A single-centre retrospective study. Acta Anaesthesiol Scand 2018. [Epub ahead of print].

35. Pasquier M, Hugli O, Paal P, et al. Hypothermia outcome prediction after extracorporeal life support for hypothermic cardiac arrest patients: The HOPE score. Resuscitation 2018;126:58-64. 